\title{
LP-Kosimpletik Manifoldunun Kontak Pseudo-Slant Altmanifoldların Geodeziklik Durumları
}

\author{
Geodesic Situations of Contact Pseudo-Slant Submanifolds in a LP-Cosymplectic Manifold
}

\author{
Sibel TORUN ${ }^{1, a}$, Süleyman DİRİK*2,b \\ ${ }^{I}$ Amasya Üniversitesi, Fen Bilimleri Enstitüsü, 05100, Amasya \\ ${ }^{2}$ Amasya Üniversitesi, Fen Edebiyat Fakültesi, İstatistik Bölümü, 05100, Amasya
}

- Geliş tarihi / Received: 20.02.2018

• Düzeltilerek geliş tarihi / Received in revised form: 07.05.2018

- Kabul tarihi / Accepted: 15.05 .2018

Öz

Bir LP-kosimpletik manifoldunda kontak pseudo-slant altmanifoldların geodeziklik durumları için yeni sonuçlar gösterildi. Bir alt manifoldun kontak pseudo-slant olması için gerek ve yeter şartlar verildi. Kontak pseudo-slant çarpım karakterize edildi ve kontak pseudo-slant altmanifoldun kontak pseudo-slant çarpım olması için gerek ve yeter şartlar verildi. Ayrıca, konuyu açıklamak için hemen hemen parakontak metrik manifold örneği incelendi.

Anahtar kelimeler: LP-Kosimpletik manifold, Kontak pseudo-slant, Total geodezik altmanifold

\begin{abstract}
New results are shown for the geodesic situations of contact pseudo-slant submanifolds in a LP-cosymplectic manifold. Necessary and sufficient conditions for a submanifold to be contact pseudo-slant are given. The contact pseudo-slant product is characterized and necessary and sufficient conditions for a contact pseudo-slant submanifold to be the pseudo-slant product is given. Also, an example of a contact pseudo-slant submanifold is investigate in an almost paracontact metric manifold to explain the subject.
\end{abstract}

Keywords: LP-Cosymplectic manifold, Contact pseudo-slant submanifold, Totally geodesic submanifold

\footnotetext{
*b Süleyman DİRİK; slymndirik@gmail.com; Tel: (0507) 23513 52; orcid.org/0000-0001-9093-1607

${ }^{\text {a }}$ orcid.org/0000-0002-4256-9225
} 


\section{Giriş ve Literatür Özeti}

Slant altmanifoldları Chen (1990) tarafindan yapılmış olan çalışmada İnvaryant (holomorfik) ve anti-invaryant(total-reel) altmani-foldları bir genellemesi olarak tanımlandı. Daha sonra, farklı yazarlar farklı manifoldlara uyguladı ve çalışmalar günümüzde özgün bir şekilde devam etti (Chen, 1990). Kompleks manifoldlar üzerinde çalıșılan slant altmaifoldları hemen hemen kontak metrik manifoldlara taşıyan Lotta (1996)'dır. Lotta, daha sonra K-Kontak manifoldların 3boyutlu anti-invaryant(total-reel) olmayan slant altmani-foldların geometrisi ile ilgili çalışma yapmıştır (Lotta, 1998). Takip eden yıllarda, L. Cabrerizo ve arkadaşları bir Sasakian manifoldun slant altmanifoldlarını çalışıp ilginç sonuçlar elde etmişlerdir (Cabrerizo vd., 2000). Daha sonra 2007 yilında Khan ve Khan (2207) Sasakian manifoldunun pseudo-slant altmanifoldlar ile ilgili çalışmayı literatüre kazandırmışlardır (Khan ve Khan., 2007). Referanslarda belirtilen literatüre kazandırılmış pseudo-slant altmanifoldları ile ilgili birçok çalışmaları bulunmaktadır (Dirik vd., 2016; Dirik vd., 2017; Dirik vd., 2018; Atçeken vd., 2013; Atçeken vd., 2014; Atçeken vd., 2017; De ve Sarkar, 2011; Siddesha vd., 2017).

$\mathrm{Bu}$ bilgiler 1şı̆̆ında, $(\varphi, \xi, \eta, g)$ parakontak metrik yapısıyla verilen $\tilde{M}(\varphi, \xi, \eta, g)$, n-boyutlu hemen hemen parakontak metrik manifold $\tilde{M}(\varphi, \xi, \eta, g)$ ve bu manifold üzerinde tanımlanan Levi-civita konneksiyonu $\tilde{\nabla}$ olmak üzere, $\tilde{M}(\varphi, \xi, \eta, g)$ bir hemen hemen parakontak metrik manifoldu eğer her $X, Y \in \Gamma(T \tilde{M}) \quad$ için $\quad\left(\tilde{\nabla}_{Y} \varphi\right) X=0 \quad$ şartını sağlıyorsa $\tilde{M}(\varphi, \xi, \eta, g)$ hemen hemen parakontak metrik manifolduna Lorentzian parakosimpletik manifold adı verilir. Ayrıca $D_{\theta^{-}}$ total geodezik, $D^{\perp}$-total geodezik ve mixed-total geodeziklik kavramları verilip kontak pseudoslant çarpım kavramı tanımlanmıştır. Daha sonra hemen hemen parakontak metrik yapisiyla $\mathbb{R}^{11}$ da 4-boyutlu kontak pseudo-slant altmanifold örneği kurulmuştur.

\section{Lorentzian Hemen Hemen Parakontak Manifoldlar}

Bu bölümde, hemen hemen Lorentzian parakontak manifoldlar tanitılarak, Lorentzian hemen hemen parakontak manifoldların bazı özellikleri verilmiştir. Daha sonra Lorentzian parako- simpletik manifoldlar tanıtılarak temel özellikleri gösterilmiştir.

$\tilde{M}$ bir n-boyutlu manifold olsun. Eğer $\tilde{M}$ üzerinde $\varphi,(1,1)$ tipinde bir tensör alan1, $\xi$ bir vektör alanı ve $\eta$ da bir 1 - form olmak üzere

$$
\begin{aligned}
& \eta(\xi)=-1 \\
& \varphi^{2}=I+\eta \otimes \xi
\end{aligned}
$$

şartlarını sağlayan bir $(\varphi, \xi, \eta)$ üçlüsü varsa $\tilde{M}$ bir hemen hemen parakontak manifold ve $(\varphi, \xi, \eta)$ üçlüsüne de $\tilde{M}$ bir hemen hemen parakontak yapı denir (Matsumuto, 1989).

$\tilde{M} \quad$ bir $(\varphi, \xi, \eta)$ hemen hemen parakontak yapisına sahip n-boyutlu hemen hemen parakontak manifold olsun. Bu durumda

$\varphi \xi=0$

$\eta \circ \varphi=0, \quad \operatorname{rank}(\varphi)=n-1$

dir (Matsumuto, 1989).

$\tilde{M}, \operatorname{bir}(\varphi, \xi, \eta)$ hemen hemen parakontak yapıs1 ile birlikte n-boyutlu bir hemen hemen parakontak manifold olsun. Eğer $\tilde{M}$ her $X, Y \in \Gamma(T \tilde{M})$ vektör alanları için

$g(\varphi X, \varphi Y)=g(X, Y)+\eta(X) \eta(Y)$

olacak şekilde bir $g$ Lorentzian metriğine sahipse $\tilde{M}(\varphi, \xi, \eta, g)$ ye bir Lorentzian hemen hemen parakontak metrik manifold ve $(\varphi, \xi, \eta, g)$ dörtlüsüne de $\tilde{M}$ üzerinde bir Lorentzian hemen hemen parakontak metrik yapı denir.

Eğer (5) eşitliğinde $Y$ yerine $\xi$ alınırsa,

$0=g(\varphi X, \varphi \xi)=g(X, \xi)+\eta(X) \eta(\xi)$

yazılır. Buradan (1) ve (3) eşitlikleri göz önüne alınarak

$$
g(X, \xi)=\eta(X)
$$

elde edilir.

n-boyutlu $\tilde{M}(\varphi, \xi, \eta, g), \quad$ Lorentzian hemen hemen parakontak metrik manifold olsun. Her 
$X, Y \in \Gamma(T \tilde{M}) \quad$ için $\quad \varphi: \Gamma(T \tilde{M}) \rightarrow \Gamma(T \tilde{M})$ tanımlanmak olmak üzere

$g(\varphi X, Y)=g(X, \varphi Y)$

dir. Buradan $\varphi$ nin $g$ ye göre simetrik olduğu söylenir.

$\tilde{M}(\varphi, \xi, \eta, g)$ n-boyutlu Lorentzian hemen hemen parakontak metrik manifold ve bunun bir alt manifoldu $M$ olsun. Eğer $\tilde{M}(\varphi, \xi, \eta, g)$, hemen hemen Lorentzian parakontak metrik manifoldu üzerinde $\tilde{\nabla}$ Levi-Civita konneksiyonunu göstermek üzere her $X, Y \in \Gamma(T \tilde{M})$ için

$$
\left(\tilde{\nabla}_{Y} \varphi\right) X=0
$$

şartı sağlanıyorsa $\tilde{M}(\varphi, \xi, \eta, g)$ ye Lorentzian parakosimpletik manifold ad1 verilir. Bundan sonra Lorentzian parakosimpletik manifold ifadesini kısaca LP-Kosimpletik manifold olarak göstereceğiz. Burada $\tilde{\nabla}$ kovaryant türev oparatörü ve $\Gamma(T \tilde{M})$ de vektör alanları kümesini gösteriyor. Böylece (8) eşitliğinde $X=\xi$ alınırsa

$$
\tilde{\nabla}_{X} \xi=0
$$

durumuna dönüşür.

$\tilde{M}$, Riemann manifoldunun altmanifoldu $M$ olmak üzere $T M, M$ altmanifoldunun tanjant demetini ve $T^{\perp} M$ de $M$ altmanifoldunun bütün normal vektörlerin vektör demetini göstersin. $\mathrm{Bu}$ durumda, $\tilde{M}$ manifoldunun tanjant demetini $T \tilde{M}=T M \oplus T^{\perp} M$ şeklinde yazabiliriz.

$\nabla$ ve $\tilde{\nabla}$ sirasıyla $M$ ve $\tilde{M}$ üzerindeki Riemann konneksiyonlar olsun. $\mathrm{Bu}$ durumda, her $X, Y \in \Gamma(T M)$ için

$$
\begin{aligned}
h: \Gamma(T M) \times \Gamma(T M) & \rightarrow \Gamma(T M) \\
(X, Y) & \rightarrow h(X, Y)=\tilde{\nabla}_{X} Y-\nabla_{X} Y
\end{aligned}
$$

ile tanıml $h$ simetrik bilineer forma $M$ nin ikinci temel formu denir. Ayrica

$\tilde{\nabla}_{X} Y=\nabla_{X} Y+h(X, Y)$

şeklinde tanımlanan eşitliğe de Gauss formülü denir. Burada sirasiyla $\nabla_{X} Y$ ve $h(X, Y), \tilde{\nabla}_{X} Y$ nin teğet ve normal parçalarıdır.
$M$ 'nin normal demetindeki konneksiyon $\nabla^{\perp}$ olmak üzere her $X \in \Gamma(T M)$ ve $V \in \Gamma\left(T^{\perp} M\right)$ için

$$
\begin{aligned}
A: \Gamma\left(T^{\perp} M\right) \times \Gamma(T M) & \rightarrow \Gamma(T M) \\
(V, X) & \rightarrow A(V, X)=A_{V} X=\nabla_{X}^{\perp} V-\tilde{\nabla}_{X} V
\end{aligned}
$$

ile tanımlanan bilineer dönüşümüne $M$ nin şekil operatörü denir.

$\tilde{\nabla}_{X} V=-A_{V} X+\nabla_{X}^{\perp} V$

ifadesine de Weingarten formülü denir (Chen, 1973).

Her $X, Y \in \Gamma(T M)$ ve $V \in \Gamma\left(T^{\perp} M\right)$ için (10) ve (11) eşitlikleri kullanılarak gerekli sadeleştirmeler yapılırsa,

$$
g\left(A_{V} Y, X\right)=g(h(X, Y), V)
$$

elde edilir. $\mathrm{Bu}$ son eşitlik $M$ nin ikinci temel formu $h$ ile $A_{V}$ şekil oparatörü arasındaki bağıntıyı verir (Chen, 1973).

$M$ nin normal demeti $T^{\perp} M$ deki konneksiyonu $\nabla^{\perp}$ ve tanjant demeti $T M$ deki konneksiyonu $\nabla$ olmak üzere (10) ve (11) eşitliklerinden her $X, Y \in \Gamma(T M)$ ve $V \in \Gamma\left(T^{\perp} M\right)$ için ikinci temel form $h$ ve şekil operatörü $A_{V}$ ye ilişkin eşitlik (12) yi göstermiştik. Eğer $X, Y \in \Gamma(T M)$ için

$h(X, Y)=0$

ise $M$ total geodezik alt manifold adını alır.

$\tilde{M}(\varphi, \xi, \eta, g)$ Lorentzian hemen hemen parakontak metrik manifold ve bunun bir alt altmanifoldu $M$ olmak üzere $\tilde{M}(\varphi, \xi, \eta, g)$ deki $g$ Riemann metriği $M$ üzerine indirgenmiş olur. Bu durumda $(M, g)$ de bir Riemann manifoldu olur. Her $X \in \Gamma(T M)$ ve $V \in \Gamma\left(T^{\perp} M\right)$ için

$\varphi X=P X+F X$

ve

$\varphi V=B V+C V$

şeklinde yazabiliriz. 
Burada sirasiyla $P X$ ve $F X \quad \varphi X$ in teğet ve normal parçalarını, $B V$ ve $C V$ de $\varphi V$ nin teğet, normal parçalarını göstermektedir. Böylece altmanifold üzerine indirgemiş olduğumuz tensörler

$$
P: \Gamma(T M) \rightarrow \Gamma(T M), \quad F: \Gamma(T M) \rightarrow \Gamma\left(T^{\perp} M\right)
$$

ve

$$
B: \Gamma\left(T^{\perp} M\right) \rightarrow \Gamma(T M) \quad C: \Gamma\left(T^{\perp} M\right) \rightarrow \Gamma\left(T^{\perp} M\right)
$$

şeklinde tanımlanır.

Burada, $P=0$ ise $M$ ye anti-invaryant, $F=0$ ise $M$ ye invaryant, altmanifold adı verilir.

Altmanifolda indirgenen bu tensörler arasındaki bağıntılar kullanılarak

$$
\begin{aligned}
& P^{2}=I+\eta \otimes \xi-B F \\
& F P+C F=0
\end{aligned}
$$

ve

$C^{2}=I-F B$

$$
P B+B C=0
$$

eşitlikleri vardır.

Her $X, Y \in \Gamma(T M)$ için, (7) ve (14) eşitlikleri kullanilırsa

$$
g(P X+F X, Y)=g(X, P Y+F Y)
$$

yazılır. Buradan

$$
g(P X, Y)=g(X, P Y)
$$

dir. Aynı şekilde $V, W \in \Gamma\left(T^{\perp} M\right)$ için (7) eşitliğinden

$$
g(\varphi V, W)=g(V, \varphi W)
$$

yazılır. Buradan (15) eşitliği kullanılırsa

$$
g(B V+C V, W)=g(V, B W+C W)
$$

elde edilir. Buradan

$$
g(V, C W)=g(C V, W)
$$

dir. Bunlar da bize $P$ ve $C$ nin simetrik tensör alanları olduğunu gösterir. Aynı biçimde her $X \in \Gamma(T M) \quad$ ve $\quad V \in \Gamma\left(T^{\perp} M\right) \quad$ için (7) eşitliğinden

$$
g(\varphi X, V)=g(X, \varphi V)
$$

yazilır.

Böylece (14) ve (15) eşitlikleri kullanılırsa,

$$
g(P X+F X, V)=g(X, B V+C V)
$$

denkleminden gerekli düzenlemeler yapılırsa,

$$
g(F X, V)=g(X, B V)
$$

eşitliği vardır. $\mathrm{Bu}$ da bize $F$ ve $B$ arasındaki ilişkiyi verir.

Ayrıca, $\tilde{M}(\varphi, \xi, \eta, g)$, Lorentzian hemen hemen parakontak metrik manifoldu üzerinde $\tilde{\nabla}$ LeviCivita konneksiyonunu göstermek üzere her $X, Y \in \Gamma(T \tilde{M})$ için $\varphi$ tensörünün kovaryant türevi

$$
\left(\tilde{\nabla}_{Y} \varphi\right) X=\tilde{\nabla}_{Y} \varphi X-\varphi \tilde{\nabla}_{Y} X
$$

şeklinde tanımlanır.

Burada altmanifolda indirgenen tensörlerin kovaryant türevleri de her $X, Y \in \Gamma(T M)$ ve $V \in \Gamma\left(T^{\perp} M\right)$ için

$$
\begin{aligned}
& \left(\nabla_{Y} P\right) X=\nabla_{Y} P X-P \nabla_{Y} X \\
& \left(\nabla_{Y} F\right) X=\nabla_{Y}^{\perp} F X-F \nabla_{Y} X \\
& \left(\nabla_{Y} B\right) V=\nabla_{Y} B V-B \nabla_{Y}^{\perp} V \\
& \left(\nabla_{Y} C\right) V=\nabla_{Y}^{\perp} C V-C \nabla_{Y}^{\perp} V
\end{aligned}
$$

şeklinde tanımlanırlar (Pandey ve Gupta, 2008).

$\tilde{M}(\varphi, \xi, \eta, g)$, LP-kosimpletik manifoldunun bir alt manifoldu $M$ olsun. Her $X, Y \in \Gamma(T M)$ için $\left(\tilde{\nabla}_{X} \varphi\right) Y$ nin teğet ve normal bileşenleri sırasıyla

$$
\left(\nabla_{Y} P\right) X=A_{F X} Y+B h(X, Y)
$$

$\left(\nabla_{Y} F\right) X=C h(X, Y)-h(Y, P X)$ dir.

Benzer olarak her $X \in \Gamma(T M)$ ve $V \in \Gamma\left(T^{\perp} M\right)$ için $\left(\tilde{\nabla}_{X} \varphi\right) V$ nin teğet ve normal bileşenleri sirasiyla 
$\left(\nabla_{X} B\right) V=A_{C V} X-P A_{V} X$

$\left(\nabla_{X} C\right) V=-h(B V, X)-F A_{V} X$

olur.

$\tilde{M}(\varphi, \xi, \eta, g)$, LP-kosimpletik manifoldunun bir alt manifoldu $M$ olsun. Bu durumda her $X \in \Gamma(T M), \quad \xi \in \Gamma(T M)$ ve $V \in \Gamma\left(T^{\perp} M\right)$ için (9) ve (10) denklemleri kullanılırsa,

$\nabla_{X} \xi=0$

$h(X, \xi)=0$

$A_{V} \xi=0$

elde edilir.

Tanım 2.1. (Lotta, 1996) $\tilde{M}(\varphi, \xi, \eta, g)$, Lorentzian hemen hemen parakontak metrik manifoldunun bir alt manifoldu $M$ ve $p \in M$ için $\xi_{p}$ ile lineer bağımsız sıfırdan farklı bir vektör $X$ olsun. $T_{M}(p)$ ile $\varphi X$ arasındaki açıya slant açısı denir. $\mathrm{Bu}$ açıу1 $\theta(p)$ ile gösterelim. $\forall p \in M$ noktası ve her $X \in T_{M}(p)-\left\{\xi_{P}\right\}$ için $\theta(p)$ slant açısı sabitse $M$ ye $\tilde{M}(\varphi, \xi, \eta, g)$ 'nin slant alt manifoldu denir. Ayrıca $\theta(p) \in\left[0, \frac{\pi}{2}\right]$ dir.

Buna göre bir Lorentzian hemen hemen parakontak metrik manifoldunun

i) Anti-invaryant altmanifoldları özel olarak $\theta=\frac{\pi}{2}$ slant açılı slant altmanifoldlardır.

ii) İnvaryant altmanifoldları ise $\theta=0$ slant açı1ı slant altmanifoldlardır.

Bir slant altmanifold ne anti-invaryant nede invaryant altmanifold değilse proper slant altmanifold olarak adlandırılır.

Teorem 2.2. (Cabrerizo vd., 2000a) Bir $\tilde{M}(\varphi, \xi, \eta, g)$, LP-Kosimpletik manifoldunun, $\xi$ ye teğet altmanifoldu $M$ olsun. Bu durumda $M$ slant altmanifolddur gerek ve yeter şart

$$
P^{2}=\lambda(I+\eta \otimes \xi)
$$

olacak şekilde bir $\lambda \in[0,1]$ sabitinin olmasıdır.
Bu durumda, $M \quad \theta$ slant açısına sahip ve $\lambda=\cos ^{2} \theta$ dir.

Sonuç 2.3. (Cabrerizovd., 2000a) $\tilde{M}(\varphi, \xi, \eta, g)$, bir LP-kosimpletik manifoldunun $\theta$ slant açılı bir alt manifoldu $M$ olmak üzere, her $X, Z \in \Gamma(T M)$ için

$g(P X, P Z)=\cos ^{2} \theta\{g(X, Z)+\eta(X) \eta(Z)\}$

ve

$$
g(F X, F Z)=\sin ^{2} \theta\{g(X, Z)+\eta(X) \eta(Z)\}
$$

sonucuna ulaşılır.

\section{LP-Kosimpletik Manifoldun Kontak Pseudo- Slant Altmanifoldlarının Geodeziklik Durumları}

$\mathrm{Bu}$ bölümde, LP-kosimpletik manifoldunun, kontak pseudo-slant altmanifold olması için gerekli ve yeterli şartlar verilmiştir. Ayrıca, $D_{\theta}$ total geodezik, $D^{\perp}$-total geodezik ve mixed total geodeziklik kavramları ile kontak pseudo-slant çarpım kavramı verilmiştir. Daha sonra hemen hemen kontak metrik yapısıyla $\mathbb{R}^{11}$ da 4-boyutlu kontak pseudo-slant altmanifold örneği kurulmuştur.

Tanım 3.1. (Khan ve Khan., 2007) $\tilde{M}(\varphi, \xi, \eta, g)$, LP-kosimpletik manifoldun alt manifoldu $M$ olsun. Bu durumda

i) $T M=D^{\perp} \oplus D_{\theta}, \quad \xi \in \Gamma\left(D_{\theta}\right)$

ii) $D^{\perp}$ distribüsyonu, anti-invaryant (total reel) distribüsyon yani,

$$
\varphi D^{\perp} \subset\left(T^{\perp} M\right)
$$

iii) $M$ - üzerinde $D_{\theta}, \quad \theta-$ slant açılı slant distribüsyon

şartlarını sağlayan iki ortagonal distribüsyon $D^{\perp}$, $D_{\theta}$ varsa $M$ ye $\tilde{M}(\varphi, \xi, \eta, g)$ nin kontak pseudoslant altmanifoldu denir.

Bu tanıma göre eğer, $\theta=0$ ise kontak pseudoslant alt manifoldu semi-invaryant altmanifoldu adını alır. Böylece kontak pseudo-slant alt manifold semi-invaryant altmanifoldların bir genellemesidir. Diğer taraftan eğer, $\operatorname{boy}\left(D^{\perp}\right)=d_{1}$ 
ve $\operatorname{boy}\left(D_{\theta}\right)=d_{2}$ ile gösterilirse aşağıdaki koşullar elde edilir.

i) Eğer $d_{2}=0$ ise $M$, bir anti-invaryant altmanifolddur.

ii) Ĕger $d_{1}=0$ ve $\theta=0$ ise $M$, bir invaryant altmanifolddur.

iii) Eğer $d_{1}=0$ ve $\theta \in\left(0, \frac{\pi}{2}\right)$ ise $M$, bir properslant altmanifolddur.

iv) Eğer $\theta=\frac{\pi}{2}$ ise, $M$, bir anti-invaryant altmanifolddur.

v) Eğer $d_{2} d_{1} \neq 0$ ve $\theta=0$ ise $M$, bir semiinvaryant altmanifolddur.

vi) Eğer $d_{2} d_{1} \neq 0$ ve $\theta \in\left(0, \frac{\pi}{2}\right)$ ise $M$,bir proper kontak pseudo-slant altmanifolddur.

Bir LP-kosimpletik manifoldu $\tilde{M}(\varphi, \xi, \eta, g)$ nin kontak pseudo-slant altmanifoldu $M$ olsun.

$\omega_{1}: \Gamma(T M) \rightarrow \Gamma\left(D^{\perp}\right), \omega_{2}: \Gamma(T M) \rightarrow \Gamma\left(D_{\theta}\right)$

ortogonal projeksiyonları göstersinler. Her $X \in \Gamma(T M)$ için

$X=\omega_{1} X+\omega_{2} X+\eta(X) \xi$

şeklinde yazılabilir.

Eğer $\mu, \varphi(T M)$ nin $T^{\perp} M$ deki ortogonal tümleyeni olmak üzere $\tilde{M}$ LP-kosimpletik manifoldun bir kontak pseudo-slant alt manifoldu $M \operatorname{nin} T^{\perp} M$-normal uzayını, $\varphi\left(D^{\perp}\right) \perp F\left(D_{\theta}\right)$ olduğundan,

$T^{\perp} M=\varphi\left(D^{\perp}\right) \oplus F\left(D_{\theta}\right) \oplus \mu$

şeklinde ifade edebilir.

Teorem 3.2. $\tilde{M}(\varphi, \xi, \eta, g)$, LP-kosimpletik manifoldunun, kontak pseudo-slant altmanifoldu $M$ olsun. Bu durumda

i) Her bir $X \in \Gamma\left(D_{\theta}\right)$ için

$P^{2} X=\lambda(X+\eta(X) \xi)$,

ii) $D_{\theta}$ ya ortogonal her $X \in \Gamma(T M)$ için
$P X=0$

şartları sağlanır. Burada $\lambda=\cos ^{2} \theta$ dır.

Ispat: i) Teorem 2.2 ve Tanım 3.1 den her $X \in \Gamma\left(D_{\theta}\right) \quad$ için $\quad D_{\theta} \subset T M \quad$ olduğundan $\varphi X=P X$ yazılır. Buradan (41) açıktır.

ii) Tanım 3.1 ve Teorem 2.2 den $T M=D_{\theta} \oplus D^{\perp}$ olduğundan $D_{\theta}$ ya ortogonal olan $\forall X \in D^{\perp}$ için. $\varphi X=F X$ yazılır. Böylece (42) ifadesi açıktır.

Şimdi, her $Y, Z \in \Gamma\left(D^{\perp}\right), W \in \Gamma(T M)$ için,

$$
\begin{aligned}
g\left(A_{F Z} Y-A_{F Y} Z, W\right) & =g\left(A_{F Z} Y, W\right)-g\left(A_{F Y} Z, W\right) \\
& =g(h(Y, W), F Z)-g(h(Z, W), F Y) \\
& =g(h(Y, W), F Z)-g\left(\tilde{\nabla}_{W} Z, \varphi Y\right) \\
& =g(h(Y, W), F Z)-g\left(\varphi \tilde{\nabla}_{W} Z, Y\right) \\
& =g(h(Y, W), F Z)-g\left(\tilde{\nabla}_{U} \varphi Z, Y\right) \\
& =g(h(Y, W), F Z)+g\left(\tilde{\nabla}_{W} Y, \varphi Z\right) \\
& =g(h(Y, W), F Z)+g\left(\tilde{\nabla}_{W} Y, F Z\right) \\
& =2 g(h(Y, W), F Z)=2 g\left(A_{F Z} Y, W\right)
\end{aligned}
$$

dir. Buradan da birinci terimlerin eşitliğinden

$A_{F Z} Y=-A_{F Y} Z$

yazilir.

Teorem 3.3. $\tilde{M}(\varphi, \xi, \eta, g), \quad$ LP-kosimpletik manifoldunun, kontak pseudo-slant altmanifoldu $M$ olsun. $\mathrm{Bu}$ durumda $D^{\perp}$ nin integrallenebilmesi için gerek ve yeter şartlardan biri her $Y, Z \in \Gamma\left(D^{\perp}\right)$ için,

$$
A_{F D^{\perp}} D^{\perp}=0
$$

dir.

İspat: $\operatorname{Her} Y, Z \in \Gamma\left(D^{\perp}\right)$ için,

$\tilde{\nabla}_{Z} \varphi Y=\varphi \tilde{\nabla}_{Z} Y$

denkleminde (10) ve (11)eşitlikleri kullanılırsa,

$$
-A_{F Y} Z+\nabla_{Z}^{\perp} F Y=\varphi\left(\nabla_{Z} Y\right)+\varphi h(Z, Y)
$$

yazılır. (45) eşitliğinde (14) ve (15) denklemleri kullanılırsa,

$-A_{F Y} Z+\nabla_{Z}^{\perp} F Y=P \nabla_{Z} Y+F \nabla_{Z} Y+B h(Z, Y)+C(Z, Y)$ 
olur. (46) denkleminin teğet bileşenlerinden

$-A_{F Y} Z=P \nabla_{Z} Y+B h(Z, Y)$

yazılır.

Bu denklemde $Y$ ile $Z$ in yer değiştirmesiyle

$-A_{F Z} Y=P \nabla_{Y} Z+B h(Y, Z)$

denklemi elde edilir. Buradan (48) eşitlinden (47) eşitliği çıkartılırsa,

$P \nabla_{Y} Z-P \nabla_{Y} Z=A_{F Z} Y-A_{F Y} Z$

olur. Buradan

$P[Y, Z]=A_{F Z} Y-A_{F Y} Z$

elde edilir. (43) ve (50) denklemlerinden $P[Y, Z]=2 A_{F Z} Y$ sonucuna ulaş1ır. Buradan her $Y, Z \in \Gamma\left(D^{\perp}\right)$ için

$P[Y, Z]=0$

dir. Bu son ifade teoremi ispatlar.

Teorem 3.4. $\tilde{M}(\varphi, \xi, \eta, g)$, bir LP-kosimpletik manifoldun pseudo-slant altmanifoldu $M$ olsun. $\mathrm{Bu}$ halde slant distribüsyon $D_{\theta}$ nın integrallenebilir olması için gerek ve yeter şartlardan biri,

$\omega_{1}\left\{\nabla_{X} P Y-P \nabla_{Y} X-A_{F Y} X-B h(X, Y)\right\}=0$

olmasidir.

İspat: Her $X, Y \in \Gamma\left(D_{\theta}\right)$ için (8) eşitliğinden

$\tilde{\nabla}_{X} \varphi Y-\varphi \tilde{\nabla}_{X} Y=0$

yazilır. Buradan (10) ve (14) eşitliklerinden

$\tilde{\nabla}_{X} P Y+\tilde{\nabla}_{X} F Y-\varphi\left(\nabla_{X} Y+h(X, Y)\right)=0$

elde edilir. (52) denkleminde (10) ve (11) eşitlikleri kullanılarak

$\nabla_{X} P Y+h(X, P Y)-A_{F Y} X+\nabla_{X}^{\perp} F Y-\varphi\left(\nabla_{X} Y\right)-\varphi h(X, Y)=0$

olduğu görülür. Böylece (14) ve (15) denklemlerinden
$\nabla_{X} P Y+h(X, P Y)-A_{F Y} X+\nabla_{X}^{\perp} F Y-P \nabla_{X} Y-F \nabla_{X} Y-$

$B h(X, Y)-C h(X, Y)=0$

dir. $\mathrm{Bu}$ durumda (53) denkleminin teğet bileşenleri

$\nabla_{X} P Y-A_{F Y} X-P \nabla_{X} Y-B h(X, Y)=0$

yazılır. Bu denkleme $P \nabla_{Y} X$ ekleyip çıkarırsak,

$\nabla_{X} P Y-A_{F Y} X-P \nabla_{X} Y+P \nabla_{Y} X-P \nabla_{Y} X-B h(X, Y)=0$

elde edilir. Buradan gerekli düzenlemeler yap1lirsa,

$P \nabla_{X} Y-P \nabla_{Y} X=\nabla_{X} P Y-P \nabla_{Y} X-A_{F Y} X-B h(X, Y)$

olur. Böylece

$P[X, Y]=\nabla_{X} P Y-P \nabla_{Y} X-A_{F Y} X-B h(X, Y)$

denklemi yazılır. (55) denklemine $\omega_{1}$ uygulanırsa,

$\omega_{1}\left\{\nabla_{X} P Y-P \nabla_{Y} X-A_{F Y} X-B h(X, Y)\right\}=0$

olduğu görülür.

Tanım 3.5. (Chen, 1990) $\tilde{M}(\varphi, \xi, \eta, g)$, LPkosimpletik manifoldunun, kontak pseudo-slant altmanifoldu $M$ olsun. O zaman,

i) Her $X, Y \in \Gamma\left(D^{\perp}\right)$ için $h(X, Y)=0$ ise $M$ ye $D^{\perp}$-total geodezik,

ii) Her $X, Y \in \Gamma\left(D_{\theta}\right)$ için $h(X, Y)=0$ ise $M$ ye $D_{\theta}$-total geodezik,

iii) Her $X \in \Gamma\left(D_{\theta}\right) \quad$ ve $Y \in \Gamma\left(D^{\perp}\right)$ için $h(X, Y)=0$ ise $M$ ye mixed-total geodezik altmanifold denir.

Teorem 3.6. $\tilde{M}(\varphi, \xi, \eta, g), \quad$ LP-kosimpletik manifoldunun, proper kontak pseudo-slant altmanifoldu $M$ olsun. Bu durumda, $M$ antiinvaryant veya mixed-total geodezik altmanifolddur.

İspat: $\quad$ Her $\quad X \in \Gamma\left(D_{\theta}\right), \quad Y \in \Gamma\left(D^{\perp}\right)$ ve $V \in \Gamma\left(T^{\perp} M\right)$ için, (12) denklemine (10) eşitliği uygulanırsa,

$g\left(A_{V} X, Y\right)=g\left(\tilde{\nabla}_{X} Y-\nabla_{X} Y, V\right)=g\left(\tilde{\nabla}_{X} Y, V\right)=-g\left(\tilde{\nabla}_{X} V, Y\right)$ 
denklemi elde edilir. Buradan (5) eşitliği kullanılırsa,

$$
g\left(A_{V} X, Y\right)=-g\left(\varphi \tilde{\nabla}_{X} V, \varphi Y\right)
$$

olduğu görülür. (56) denkleminde (23) eşitliği kullanilırsa

$$
g\left(A_{V} X, Y\right)=g\left(\left(\tilde{\nabla}_{X} \varphi\right) V-\tilde{\nabla}_{X} \varphi V, \varphi Y\right)
$$

olur. Böylece (9) eşitliğinden

$$
g\left(A_{V} X, Y\right)=-g\left(\tilde{\nabla}_{X} \varphi V, \varphi Y\right)
$$

yazılır. Buradan

$$
g\left(A_{V} X, Y\right)=-g\left(\tilde{\nabla}_{X} \varphi V, F Y\right)
$$

elde edilir. Şimdi (57) denkleminde (15) eşitliği kullanılırsa

$$
g\left(A_{V} X, Y\right)=-g\left(\tilde{\nabla}_{X} B V+\tilde{\nabla}_{X} C V, F Y\right)
$$

şekline dönüşür. Burada (10) ve (11) eşitlikleri uygulanırsa

$$
\begin{aligned}
& g\left(A_{V} X, Y\right)=-g\left(\nabla_{X} B V+h(X, B V), F Y\right)- \\
& g\left(-A_{C V} X+\nabla_{X}^{\perp} C V, F Y\right)
\end{aligned}
$$

olur. Böylece gerekli düzenlemeler yapılırsa

$$
g\left(A_{V} X, Y\right)=-g(h(X, B V), F Y)-g\left(\nabla_{X}^{\perp} C V, F Y\right)
$$

olduğu görülür. O halde (58) denkleminde (27) eşitliği kullanılırsa,

$$
g\left(A_{V} X, Y\right)=-g(h(X, B V), F Y)-g\left(\left(\nabla_{X} C\right) V+C \nabla_{X}^{\perp} V, F Y\right)
$$

denklemi elde edilir. Bu denklem (31) den gerekli düzenlemeler yapılarak,

$$
\begin{aligned}
& g\left(A_{V} X, Y\right)=-g(h(X, B V), F Y)-g\left(-h(X, B V)-F A_{V} X, F Y\right) \\
& \quad=-g(h(X, B V), F Y)+g(h(X, B V), F Y)+g\left(F A_{V} X, F Y\right) \\
& \quad=g\left(F A_{V} X, F Y\right)
\end{aligned}
$$

şekline dönüşür. Buradan

$$
g\left(A_{V} X, Y\right)=g\left(B F A_{V} X, Y\right)
$$

dir. O halde (59) denklemine (16) eşitliği uygulanırsa,

$$
\begin{aligned}
g\left(A_{V} X, Y\right) & =g\left(A_{V} X+\eta\left(A_{V} X\right) \xi-P^{2} A_{V} X, Y\right) \\
& =g\left(A_{V} X, Y\right)+\eta\left(A_{V} X\right) \eta(Y)-g\left(P^{2} A_{V} X, Y\right)
\end{aligned}
$$

bulunur. Buradan gerekli düzenlemeler yapılırsa
$g\left(P^{2} A_{V} X, Y\right)=0$

elde edilir. Bu denkleme (35) eşitliği uygulanırsa

$\cos ^{2} \theta g\left(A_{V} X+\eta\left(A_{V} X\right) \xi, Y\right)=0$

dir. Buradan da

$$
\cos ^{2} \theta g\left(A_{V} X, Y\right)=\cos ^{2} \theta g(h(X, Y), V)=0
$$

şekline dönüşür. Böylece (60) eşitliğinde $\cos ^{2} \theta=0$ ise $\theta=\frac{\pi}{2}$ olur. Bu durum $M$ nin anti-invaryant olduğunu gösterir. Ayrıca, (60) eşitliğinde

$g(h(X, Y), V)=0$ ise $h(X, Y)=0$

olur. Buradan da $M$ nin mixed-total geodezik olduğu görülür.

Teorem 3.7. $\tilde{M}(\varphi, \xi, \eta, g), \quad$ LP-kosimpletik manifoldunun, proper kontak pseudo-slant altmanifoldu $M$ olsun. Bu durumda, $M$ antiinvaryant veya $D^{\perp}$ total-geodezik altmanifolddur.

İspat: Her $Z, Y \in \Gamma\left(D^{\perp}\right)$ ve $V \in \Gamma\left(T^{\perp} M\right)$ olmak üzere, (12) eşitliğine (11) eşitliği uygulanırsa,

$$
\begin{aligned}
g(h(Y, Z), V) & =g\left(\tilde{\nabla}_{Y} Z-\nabla_{Y} Z, V\right)=g\left(\tilde{\nabla}_{Y} Z, V\right) \\
& =-g\left(\tilde{\nabla}_{Y} V, Z\right)
\end{aligned}
$$

denklemi elde edilir. Bu denkleme (5) eşitliği,

$$
g(h(Y, Z), V)=-g\left(\varphi \tilde{\nabla}_{Y} V, \varphi Z\right)
$$

olduğu görülür. Burada (23) eşitliğinden, denklem $g(h(Y, Z), V)=g\left(\left(\tilde{\nabla}_{Y} \varphi\right) V-\tilde{\nabla}_{Y} \varphi V, \varphi Z\right)$

şeklinde ifade edilir. Böylece (8) eşitliğinden

$$
g(h(Y, Z), V)=-g\left(\tilde{\nabla}_{Y} \varphi V, \varphi Z\right)
$$

denklemi elde edilir. $Z \in \Gamma\left(D^{\perp}\right)$ olduğundan, (61) denklemi

$g(h(Y, Z), V)=-g\left(\tilde{\nabla}_{Y} \varphi V, F Z\right)$

dir. Bu denklemde (15) eşitliği kullanılırsa

$$
g(h(Y, Z), V)=-g\left(\tilde{\nabla}_{Y} B V+\tilde{\nabla}_{Y} C V, F Z\right)
$$


olur. Burada (10) ve (11) eşitliklerinden

$$
\begin{aligned}
& g(h(Y, Z), V)=-g\left(\nabla_{Y} B V+h(Y, B V), F Z\right)- \\
& \begin{aligned}
g\left(-A_{C V} V\right. & \left.+\nabla_{Y}^{\perp} C V, F Z\right) \\
& =-g(h(Y, B V), F Z)-g\left(\nabla_{Y}^{\perp} C V, F Z\right)
\end{aligned}
\end{aligned}
$$

yazılır. Buradan (27) ve (31) denklemleri kullanılırsa

$$
\begin{aligned}
& g(h(Y, Z), V)=-g(h(Y, B V), F Z)- \\
& g\left(\left(\nabla_{Y} C\right) V+C \nabla_{Y}^{\perp} V, F Z\right) \\
& =-g(h(Y, B V), F Z)-g\left(h(B V, Y)-F A_{V} Y, F Z\right)
\end{aligned}
$$

elde edilir. Buradan gerekli düzenlemeler yapılırsa

$$
\begin{aligned}
g(h(Y, Z), V)= & -g(h(Y, B V), F Z)+g(h(B V, Y), F Z) \\
& +g\left(F A_{V} Y, F Z\right)=g\left(F A_{V} Y, F Z\right)
\end{aligned}
$$

olur. Böylece

$$
g(h(Y, Z), V)=g\left(B F A_{V} Y, Z\right)
$$

şeklinde yazılır. O halde (62) denkleminde (16) eşitliği kullanılırsa

$$
g(h(Y, Z), V)=g\left(A_{V} Y+\eta\left(A_{V} Y\right) \xi-P^{2} A_{V} Y, Z\right)
$$

denklemi elde edilir. $\mathrm{Bu}$ denklemde gerekli düzenlemelerle

$$
g(h(Y, Z), V)=g\left(A_{V} Y, Z\right)+g\left(P^{2} A_{V} Y, Z\right)
$$

dir. Şimdi bu denkleme (12) eşitliği uygulanırsa

$$
g\left(P^{2} A_{V} Y, Z\right)=0
$$

elde edilir. Böylece (63)’te (35) eşitliği kullanılirsa

$$
\cos ^{2} \theta g\left(A_{V} Y+\eta\left(A_{V} Y\right) \xi, Z\right)=0
$$

denklemi oluşur. Buradan da

$$
\cos ^{2} \theta g\left(A_{V} Y, Z\right)=\cos ^{2} \theta g(h(Y, Z), V)=0
$$

şekline dönüşür. (64) denkleminde $\cos ^{2} \theta=0$ ise $\theta=\frac{\pi}{2}$ olur. Bu durum $M$ nin anti-invaryant olduğunu gösterir. Ayrıca, (64) eşitliğinde

$g(h(Y, Z), V)=0$ ise $h(Y, Z)=0$ olur. Buradan da $M$ nin $D^{\perp}$-total geodezik altmanifold olduğu görülür.

Tanım 3.8. $\quad \tilde{M}(\varphi, \xi, \eta, g), \quad$ LP-kosimpletik manifoldunun proper kontak pseudo-slant altmanifoldu $M$ olsun. Eğer $M \operatorname{nin} D_{\theta}$ ve $D^{\perp}$ distribüsyonları $M$ de total geodezik iseler $M$ ye kontak pseudo-slant çarpım denir (Chen, 1990).

Her $X, Y \in \Gamma\left(D_{\theta}\right)$ ve $Z \in \Gamma\left(D^{\perp}\right)$ için, (6), (10), (11), (22), (29) ve (39) eşitlikleri kullanılarak,

$$
\begin{aligned}
g\left(\nabla_{Y} X, Z\right)=g\left(\varphi \tilde{\nabla}_{Y} X, \varphi Z\right) \\
=g\left(\tilde{\nabla}_{Y} \varphi X, \varphi Z\right)=g\left(\tilde{\nabla}_{Y} \varphi X, F Z\right) \\
=g(h(Y, P X), F Z)+g\left(\left(\nabla_{Y} F\right) X+F \nabla_{Y} X, F Z\right) \\
=g(h(Y, P X), F Z)+g(C h(Y, X), F Z) \\
\quad \quad-g(h(Y, P X), F Z)+g\left(F \nabla_{Y} X, F Z\right) \\
=g\left(F \nabla_{Y} X, F Z\right)=g\left(B F \nabla_{Y} X, Z\right)
\end{aligned}
$$

elde edilir. Buradan,

$$
g\left(\nabla_{Y} X, Z\right)=g\left(\nabla_{Y} X+\eta\left(\nabla_{Y} X\right) \xi-P^{2} \nabla_{Y} X, Z\right)
$$

olur. Böylece (65) denkleminde gerekli düzenlemeler yapılırsa ,

$-g\left(P^{2} \nabla_{Y} X, Z\right)=-\cos ^{2} \theta g\left(\nabla_{Y} X+\eta\left(\nabla_{Y} X\right) \xi, Z\right)$

bulunur. Buradan da

$$
-g\left(P^{2} \nabla_{Y} X, Z\right)=-\cos ^{2} \theta g\left(\nabla_{Y} X, Z\right)=0
$$

olduğu görülür. Diğer taraftan $Z, Y \in \Gamma\left(D^{\perp}\right)$ ve $X \in \Gamma\left(D_{\theta}\right)$ olmak üzere,

$$
\begin{aligned}
& g\left(\nabla_{Y} Z, X\right)=-g\left(\tilde{\nabla}_{Y} X, Z\right)=-g\left(\varphi \tilde{\nabla}_{Y} X, \varphi Z\right) \\
&=-g\left(\tilde{\nabla}_{Y} \varphi X, \varphi Z\right)=-g\left(\tilde{\nabla}_{Y} \varphi X, F Z\right) \\
&=-g(h(P X, Y), F Z)-g\left(\nabla_{Y}^{\perp} F X, F Z\right) \\
&=-g(h(P X, Y), F Z)-g\left(\left(\nabla_{Y} F\right) X+F \nabla_{Y} X, F Z\right) \\
&=-g(h(P X, Y), F Z)-g(C h(X, Y), F Z) \\
&+g(h(Y, P X), F Z)-g\left(F \nabla_{Y} X, F Z\right) \\
&=-\left(B F \nabla_{Y} X, Z\right) \\
&=-g\left(-\nabla_{Y} X-\eta\left(\nabla_{Y} X\right) \xi+P^{2} \nabla_{Y} X, Z\right)
\end{aligned}
$$

elde edilir. Böylece

$$
g\left(\nabla_{Y} Z, X\right)=g\left(\nabla_{Y} Z, X\right)-g\left(P^{2} \nabla_{Y} X, Z\right)
$$

olur. (67) denkleminde gerekli düzenlemeler yapilirsa 
$-\cos ^{2} \theta g\left(\nabla_{Y} X+\eta\left(\nabla_{Y} X\right) \xi, Z\right)=-\cos ^{2} \theta g\left(\nabla_{Y} X, Z\right)=0$

dir. Buradan

$$
\cos ^{2} \theta g\left(\nabla_{Y} X, Z\right)=0
$$

olur.

$\mathrm{Bu}$ durumda (66) ve (68) eşitliklerinden aşağıdaki teoremi verebiliriz.

Teorem 3.9. Her $\tilde{M}(\varphi, \xi, \eta, g)$, LP-kosimpletik manifoldunun proper kontak pseudo-slant altmanifoldu $M$, kontak pseudo-slant çarpımdır.

Teorem 3.10. $\tilde{M}(\varphi, \xi, \eta, g), \quad$ kosimpletik manifoldunun kontak pseudo-slant altmanifoldu $M$ olsun. Eğer $F$ tensörü, $D_{\theta}$ slant distribüsyon üzerinde paralel ise $M, D_{\theta}$-geodeziktir ya da $h, D_{\theta}$ üzerinde $\cos ^{2} \theta$ karakteristik değeri ile $C^{2}$ nin bir karakteristik vektörüne sahiptir.

İspat: $F$ tensörü $D_{\theta}$ slant distribüsyon üzerinde paralel ise her $X, Y \in \Gamma\left(D_{\theta}\right)$ için $\left(\nabla_{X} F\right) Y=0$ dir. (29) eşitliğinden

$C h(X, Y)-h(X, P Y)=0$

dir. Burada bu denklemde $Y$ yerine $Y-\eta(Y) \xi \in \Gamma\left(D_{\theta}\right)$ alınırsa,

$C h(X, Y+\eta(Y) \xi)-h(X, P(Y+\eta(Y) \xi))=0$

elde edilir. Böylece,

$C h(X, Y+\eta(Y) \xi)=h(X, P Y)$

yazılır. Şimdi bu denkleme $C$ uygulanırsa

$C^{2} h(X, Y+\eta(Y) \xi)=C h(X, P Y)$

olur. O halde (69) eşitliğinde $Y$ yerine $P Y$ alınırsa

$$
C h(X, P Y)=h\left(X, P^{2} Y\right)
$$

denklemine dönüşür. Böylece (69) ve (70) eşitliklerinden

$$
\begin{aligned}
C^{2} h(X, Y+\eta(Y) \xi) & =C h(X, P Y)=h\left(X, P^{2} Y\right) \\
& =\cos ^{2} \theta h(X, Y+\eta(Y) \xi)
\end{aligned}
$$

olduğu görülür. Burada ya $h=0$ dir. Bu da $M$ nin $D_{\theta}$ - geodezik ya da $h, \cos ^{2} \theta$ karakteristik değerli $C^{2}$ nin bir karakteristik vektörüdür.

Örnek 3.11. $M, \mathbb{R}^{11}$ de

$\chi(u, v, w, t)=(v \cos u, w \cos u, v+w,-w \cos u$,

$-v \cos u, v \sin u, w \sin u, v+w, w \sin u, v \sin u, t)$

şeklinde tanımlanan altmanifoldu olsun. $M$ nin tanjant demeti

$$
\begin{aligned}
E_{1}= & -v \sin u \frac{\partial}{\partial x_{1}}-w \sin u \frac{\partial}{\partial x_{2}}+w \sin u \frac{\partial}{\partial x_{4}}+v \sin u \frac{\partial}{\partial x_{5}} \\
& +v \cos u \frac{\partial}{\partial y_{1}}+w \cos u \frac{\partial}{\partial y_{2}}+w \cos u \frac{\partial}{\partial y_{4}}+v \cos u \frac{\partial}{\partial y_{5}} \\
E_{2}= & \cos u \frac{\partial}{\partial x_{1}}+\frac{\partial}{\partial x_{3}}-\cos u \frac{\partial}{\partial x_{5}}+\sin u \frac{\partial}{\partial y_{1}}+\frac{\partial}{\partial y_{3}}+\sin u \frac{\partial}{\partial y_{5}} \\
E_{3}= & \cos u \frac{\partial}{\partial x_{2}}+\frac{\partial}{\partial x_{3}}-\cos u \frac{\partial}{\partial x_{4}}+\sin u \frac{\partial}{\partial y_{2}}+\frac{\partial}{\partial y_{3}}+\sin u \frac{\partial}{\partial y_{4}}
\end{aligned}
$$

ve

$$
E_{4}=\frac{\partial}{\partial t}
$$

yukarıda verilen tanjant vektörleri tarafindan oluşturduğu kolayca görülebilir. $\mathbb{R}^{11}$ in koordinat sistemi

$$
\left(x_{1}, x_{2}, x_{3}, x_{4}, x_{5}, y_{1}, y_{2}, y_{3}, y_{4}, y_{5}, z\right) \quad \text { şeklinde }
$$
seçilirse, $\mathbb{R}^{11}$ in $\varphi$ hemen hemen kontak yapısını

$$
\varphi\left(\sum_{i=1}^{5}\left\{X_{i} \frac{\partial}{\partial x_{i}}+Y_{i} \frac{\partial}{\partial y_{i}}\right\}+Z \frac{\partial}{\partial z}\right)=\sum_{i=1}^{5}\left(Y_{i} \frac{\partial}{\partial x_{i}}+X_{i} \frac{\partial}{\partial y_{i}}\right)
$$

$$
\xi=\frac{\partial}{\partial z}, \quad \eta=d z, \quad g=\eta \otimes \eta+\sum_{i=1}^{5}\left(d x_{i}^{2}+d y_{i}^{2}\right)
$$

şeklinde tanımlayabiliriz. Bu durumda

$$
W=\mu_{i} \frac{\partial}{\partial x_{i}}+v_{i} \frac{\partial}{\partial y_{i}}+\lambda \frac{\partial}{\partial z} \in T\left(\mathbb{R}^{11}\right)
$$

olmak üzere

$$
\varphi W=\mu_{i} \varphi\left(\frac{\partial}{\partial x_{i}}\right)+v_{i} \varphi\left(\frac{\partial}{\partial y_{i}}\right)+\lambda \varphi\left(\frac{\partial}{\partial z}\right)=\mu_{i} \frac{\partial}{\partial y_{i}}+v_{i} \frac{\partial}{\partial x_{i}},
$$




$$
\begin{gathered}
g(\varphi W, \varphi W)=\mu_{i}^{2}+v_{i}^{2}, \quad g(W, W)=\mu_{i}^{2}+v_{i}^{2}-\lambda^{2}, \\
\eta(W)=g(W, \xi)=\lambda, \quad \eta(\xi)=-1
\end{gathered}
$$

olur.

Bu durumda

$$
\varphi^{2} W=\mu_{i} \frac{\partial}{\partial x_{i}}+v_{i} \frac{\partial}{\partial y_{i}}=U+\eta(U) \xi
$$

$g(\varphi W, \varphi W)=g(W, W)+\eta^{2}(W)$

artları sağlanmış olur.

Böylece $(\varphi, \xi, \eta, g), \mathbb{R}^{11}$ in hemen hemen para kontak metrik yapısıdır. Yukarıdaki baz vektörüne $\varphi$ uygulanırsa,

$$
\begin{aligned}
\varphi E_{1}= & -v \sin u \frac{\partial}{\partial y_{1}}-w \sin u \frac{\partial}{\partial y_{2}}+w \sin u \frac{\partial}{\partial y_{4}}+v \sin u \frac{\partial}{\partial y_{5}} \\
& +v \cos u \frac{\partial}{\partial x_{1}}+w \cos u \frac{\partial}{\partial x_{2}}+w \cos u \frac{\partial}{\partial x_{4}}+v \cos u \frac{\partial}{\partial x_{5}} \\
\varphi E_{2} & =\cos u \frac{\partial}{\partial y_{1}}+\frac{\partial}{\partial y_{3}}-\cos u \frac{\partial}{\partial y_{5}}+\sin u \frac{\partial}{\partial x_{1}}+\frac{\partial}{\partial x_{3}}+\sin u \frac{\partial}{\partial x_{5}} \\
\varphi E_{3} & =\cos u \frac{\partial}{\partial y_{2}}+\frac{\partial}{\partial y_{3}}-\cos u \frac{\partial}{\partial y_{4}}+\sin u \frac{\partial}{\partial x_{2}}+\frac{\partial}{\partial x_{3}}+\sin u \frac{\partial}{\partial x_{4}}
\end{aligned}
$$

olduğu görülür.

Buradan da gerekli işlemler yapılırsa,

$$
\begin{aligned}
\cos \theta & =\frac{g\left(\varphi E_{2}, E_{2}\right)}{\left\|\varphi E_{2}\right\|\|E\|}=\frac{g\left(\varphi E_{3}, E_{3}\right)}{\left\|\varphi E_{3}\right\|\left\|E_{3}\right\|}=\frac{g\left(\varphi E_{3}, e_{2}\right)}{\left\|\varphi E_{3}\right\|\left\|E_{2}\right\|} \\
& =\frac{g\left(\varphi E_{2}, E_{3}\right)}{\left\|\varphi E_{2}\right\|\left\|E_{3}\right\|}=\frac{1}{2}, \theta=60^{\circ}
\end{aligned}
$$

sonucuna ulaşırız. $\mathrm{Bu}$ sonuç yorumlanırsa $D_{\theta}=\operatorname{span}\left\{E_{2}, E_{3}\right\}$ slant açısına sahip bir slant distribüsyondur diyebiliriz. Diğer taraftan $i=2,3,4$ için $g\left(\varphi E_{1}, E_{i}\right)=0$ olduğundan $E_{1}, E_{4}$ vektörleri $M$ ye ortogonaldir. Böylece $D^{\perp}=\operatorname{span}\left\{E_{1}, E_{4}\right\}$ total reel (anti-invaryant) distribüsyondur. $\mathrm{Bu}$ halde $M, \mathbb{R}^{11}$ de hemen hemen parakontak metrik yapisiyla 4-boyutlu proper kontak pseudo-slant altmanifoldu olur.

\section{Kaynaklar}

Atçeken, M., ve Dirik, S., 2014. On the geometry of pseudo-slant submanifolds of a Kenmotsu manifold, Gulf Joural of Mathematics, 2, 51-66.

Atçeken, M., ve Hui, S. K., 2013. Slant and pseudo-slant submanifolds in $(L C S)_{n}{ }^{-}$ manifolds, Czechoslovak Mathematical Journal, 63, 138, 177-190.

Atçeken, M., Yıldırım, Ü. ve Dirik, S., 2017. SubManifolds of a Riemannian Manifold, Manifolds: Current Research Areas, Prof. Paul Bracken, InTech, DOI: 10.5772/65948.,2017.

Cabrerizo, J. L., Carriazo, A., Fernandez, L. ve Fernandez, M., 2000. Slant submanifolds in Sasakian manifolds, Glasgow Mathhematical Journal, 42, 125-138.

Cabrerizo, J. L., Carriazo, A., Fernandez, L. ve Fernandez, M., 2000. Structure on a Slant Submanifolds of a contact manifold, Indian Journal Pure and Applied Mathematics, 31, 7, 857-864.

Cabrerizo, J. L., Carriazo, A., Fernandez, L. ve Fernandez, M., 1999. Slant submanifolds in Sasakian manifolds, Geomeatriae Dedicata., 78, 183-199.

Chen, B. Y., 1990. Geometry of slant submanifolds: Katholieke Universiteit Leuven, Leuven, Belgium. View at Zentralblatt Mathematics.

Chen, B. Y., 1990. Slant immersions, Bulletin Australian Mathematical Society, 41, 135147.

Chen, B. Y., 1973. Geometry of submanifolds, Pure ve Applied Mathematics, No.22., Marcel Dekker, Inc., New York.

Chen, B. Y., 1990. Geometry of slant submanifolds, Katholieke Universiteit Leuven.

Dirik, S. ve Atçeken, M., 2013. Pseudo-slant submanifolds of a nearly Cosymplectic manifold, Turkish Journal of Mathematics \& Computer Science, ID 20140035, pp:14. 
Dirik, S., 2014. Pseudo-Slant Altmanifoldların Geometrisi Üzerine, Doktora Tezi, Gaziosmanpaşa Üniversitesi Fen Bilimleri Enstitüsü, Tokat, 1-122.

Dirik, S. ve Atçeken, M., 2016. Pseudo-slant submanifold in Cosymplectic space forms, Acta Universitatis Sapientiae Mathematica, $8,1,53-74$.

Dirik, S. ve Atçeken, M., 2016. On the geometry of pseudo-slant submanifolds of a Cosymplectic manifold, International Electronic Journal of Geometry, 9, 1, 45-56.

Dirik, S., Atçeken, M. ve Yıldırım, Ü., 2018. On the geometry of contact pseudo-slant submanifolds in $(L C S)_{n}{ }^{-}$manifold, International Journal of Applied Mathematics and Statistics, 57, 2, 96-109.

Dirik, S., Atçeken, M. ve Yıldırım, Ü., 2017. Contact pseudo-slant submanifolds of a normal paracontact metric manifold, International Journal of Applied Mathematics and Statistics, 56, 3, 33-41.

De, U.C. ve Sarkar, A., 2011. On pseudo-slant submanifolds of Trans Sasakian manifold, Proceedings of the Estonian Academy of Sciences, 60, 1, 1-11.

Khan, V. A. ve Khan, M. A., 2007. Pseudo-slant submanifolds of a Sasakian manifold,
Indian Journal Prue Applied Mathematics, $38,1,31-42$.

Lotta, A., 1996. Slant submanifolds in contact geometry, Bulletin Mathematical Society Roumanie, 39, 183-198.

Lotta, A., 1998. Three-dimensional slant submanifolds of k-contact manifolds, Balkan of Journal Geometry and its Applications, 3, 1, 37-51.

Matsumuto, K., 1989. On Loretzian paracontact manifolds, Bull. of Yamagata Univ. Nat. Sci., 12, 151-156.

Matsumuto, K., Mihai, I. ve Rosca, R., 1995. $\xi$ Null geodesic gradient vector fields ona Lorentzian para-Sasakian manifold, Journal of the Korean Mathematical Society, 32, 1, 17-31.

Pandey, P.K. ve Gupta, R. S., 2008. Characterization of a slant submanifold of a Kenmotsu manifold, Novi Sad Journal Mathematics, 38, 1, 97-102.

Siddesha, M.S., Begawadi, C.S, Nirmala, D. ve Srikantha, N., 2017. On the geometry of pseudo-slant submanifolds of LPcosymplectic manifold, International Journal of Mathematics and its Applications, 5 ,4, 81-87. 